\title{
Non-intrusive model reduction for a 3D unstructured mesh control volume finite element reservoir model and its application to fluvial channels
}

\author{
D. Xiao
}

Applied Modelling and Computation Group, Department of Earth Science and Engineering, Imperial College London, Prince Consort Road, SW7 2BP, London, UK and Data Assimilation Lab, Data Science Institute, Imperial College London, UK Email: dh.xiao@imperial.ac.uk

\section{F. Fang and C.C. Pain}

Applied Modelling and Computation Group, Department of Earth Science and Engineering, Imperial College London,

Prince Consort Road, SW7 2BP, London, UK

Email: f.fang@imperial.ac.uk

Email: c.pain@imperial.ac.uk

\section{I.M. Navon}

Department of Scientific Computing, Florida State University,

Tallahassee, FL, 32306-4120, USA

Email: inavon@fsu.edu

\section{P. Salinas}

Applied Modelling and Computation Group, Department of Earth Science and Engineering, Imperial College London, Prince Consort Road, SW7 2BP, London, UK Email: pablo.salinas@imperial.ac.uk 


\title{
Z. Wang ${ }^{*}$
}

School of Earth Sciences and Engineering,

Sun Yat-Sen University, China

Email: wzhengh@mail.sysu.edu.cn

*Corresponding author

\begin{abstract}
A non-intrusive model reduction computational method using hypersurfaces representation has been developed for reservoir simulation and further applied to 3D fluvial channel problems in this work. This is achieved by a combination of a radial basis function (RBF) interpolation and proper orthogonal decomposition (POD) method. The advantage of the method is that it is generic and non-intrusive, that is, it does not require modifications to the original complex source code, for example, a 3D unstructured mesh control volume finite element (CVFEM) reservoir model used here. The capability of this non-intrusive reduced order model (NIROM) based on hypersurfaces representation has been numerically illustrated in a horizontally layered porous media case, and then further applied to a 3D complex fluvial channel case. By comparing the results of the NIROM against the solutions obtained from the high fidelity full model, it is shown that this NIROM results in a large reduction in the CPU computation cost while much of the details are captured. [Received: May 19, 2017; Accepted: December 1, 2017]
\end{abstract}

Keywords: radial basis function; RBF; proper orthogonal decomposition; POD; reservoir; hypersurface; 3D fluvial channel.

Reference to this paper should be made as follows: Xiao, D., Fang, F., Pain, C.C., Navon, I.M., Salinas, P. and Wang, Z. (2018) 'Non-intrusive model reduction for a $3 \mathrm{D}$ unstructured mesh control volume finite element reservoir model and its application to fluvial channels', Int. J. Oil, Gas and Coal Technology, Vol. 19, No. 3, pp.316-339.

Biographical notes: D. Xiao is a Research Associate in the Imperial College London from October 2016 up to present. He develop reduced order model for EPSRC project: managing air for green inner cities (MAGIC). He has a $\mathrm{PhD}$ in the Imperial College London, from October 2013 to February 2016. His research interests are non-intrusive model reduction for multiphase, fluids-solids coupling, air pollution problems, non-intrusive reduced order modelling for Navier-Stokes equations, fluid-structure interactions and multiphase flows in porous media. He is awarded as the Best Research Award. He has also an MSc in the China University of Geosciences from 2006-2008 in 3D Gravity and Magnetic Inversion (finished one year earlier than anyone else, Top 1) and BSc in the Wuhan University of Science and Technology, Computer Sciences (Top 5).

F. Fang is a Senior Research Fellow in 2017 in the Department of Earth Science and Engineering, Imperial College London. He is also Research Fellow form 2010-present in the Department of Earth Science and Engineering, Imperial College London. He is a Research Associate form 2009-2010 in the joint position at the Imperial College London and University of Karlsruhe and Research Associate from 2002-2009 in the Department of Earth Science and Engineering, Imperial College London. 
C.C. Pain is a Professor in the Department of Earth Science and Engineering, Imperial College London from 2005-present. He is also the Head of the Applied Computation and Modelling Group (AMCG) at Imperial College London. He holds an MSc in 1989 at Computational Fluid Dynamics (University of Exeter - Mathematics and Engineering Departments). And then he worked as a Research Associate and then Research Fellow in the Department of Earth Science and Engineering, Imperial College London from 1993 and 1995 respectively. He has his MSc at Cranfield University in 1989, and his Bachelor at University of Reading from 1985 to 1988.

I.M. Navon is a Professor in the Department of Mathematics, Florida State University from 1991-present. He is an Associate Professor in the Department of Mathematics, Florida State University, Tallahassee, Florida, from 1987 to 1990. He holds a PhD of Applied Mathematics at the University of Witwatersrand, Johannesburg, South Africa from 1977 to 1979 . He holds an MSc of Meteorology in Hebrew University of Jerusalem from 1968 to 1971.

P. Salinas is a Post-Doctoral Research Associate in the Imperial College London from 2013-present. He has a PhD in the Computational Mechanics, University of Zaragoza from 2011-2013. He has a Master in Applied Mechanics, University of Zaragoza from 2011-2012 and Industrial Engineer, University of Zaragoza from 2004-2010.

Z. Wang is an Associate Professor in the School of Earth Sciences and Engineering, Sun Yat-Sen University from 2006-present. He holds a $\mathrm{PhD}$ at China University of Geosciences (Wuhan) from 2001 to 2004. He holds a Master's degree at China University of Geosciences (Wuhan) from 1998-2001. He holds a Bachelor at China University of Geosciences (Wuhan) from 1992-1996.

\section{Introduction}

Reservoir simulation is very important and has a wide range of applications, from ground-water production to radioactive waste and the extraction of oil and gas from the subsurface. 3D reservoir modelling provides more details for the multiphase flows in porous media. However, the computational cost of 3D reservoir simulations is high. The non-intrusive reduced order modelling technique presented here is capable of resolving $3 \mathrm{D}$ reservoir modelling problems while avoiding the high computational cost.

The reduced order modelling technique has been shown to possess a powerful capability of representing the large dynamical systems using only a small number of reduced order basis functions. Among the model reduction methods, the POD approach is the most widely used. This method extracts the most energetic parts of the system through snapshots method, and then constructs optimal basis functions. The POD method has been successfully applied to numerous research fields. In geophysical fluid dynamics it is referred to as empirical orthogonal functions (EOF) (Jolliffe, 2002), in signal analysis it is termed as Karhunen-Love method (Fukunaga, 1990) and in statistics it is called the principal component analysis (PCA) method (Pearson, 1901). The proper orthogonal decomposition (POD) technique has also been applied to ocean models (Xiao et al., 2014; Fang et al., 2013; Stefanescu and Navon, 2013; Stefanescu et al., 2014; Du et al., 2013), air pollution modelling (Fang et al., 2014), data assimilation (Daescu and 
Navon, 2008; Altaf, 2011; Chen et al., 2011; Chen et al., 2012; Cao et al., 2007) and mesh optimisation (Fang et al., 2010).

The most widely used method of deriving a reduced order model (ROM) is through the combination of POD approach and Galerkin projection. The computational efficiency of the ROM can be increased by several orders of magnitude by projecting the 3D dynamical physical system onto a reduced space. However, this method suffers from numerical instability (Franca and Frey, 1992). Various methods have been proposed to overcome or improve the stability issue of the POD/Galerkin projection method, including nonlinear Petrov-Galerkin (Fang et al., 2013; Xiao et al., 2013), regularisation (Jafarpour and Sabetghadam, 2012), sub grid-scale modelling, calibration (Bou-Mosleh et al., 2011; Serpas et al., 2011) and Fourier expansion (Willcox and Megretski, 2003). Another issue that arises in the ROMs is the efficient treatment of nonlinear terms in the partial differential equations (PDEs). A number of efficient nonlinear treatment methods have been presented, for example, Gappy POD (Willcox, 2006), the empirical interpolation method (EIM) (Barrault et al., 2004) and its discrete version discrete empirical interpolation method (DEIM) (Chaturantabut and Sorensen, 2010), residual DEIM (Xiao et al., 2014), Petrov-Galerkin projection method (Bou-Mosleh et al., 2011), Gauss-Newton with approximated tensors (GNAT) method (Carlberg et al., 2013) and the quadratic expansion method (Du et al., 2013; Fang et al., 2009). However, these methods are still dependent on the source code of the original physical system. In most cases the source code describing the full physical system has to be modified in order to form the ROM.

To circumvent these disadvantages, more recently, non-intrusive methods have been introduced into model reduction, which do not require the knowledge of the governing equations and the original source code (Han, 2012). Han (2012) proposed a nonintrusive model reduction method based on black-box stencil interpolation method and machine learning method. Walton et al. (2013) proposed a non-intrusive reduced order technique for unsteady fluid flow using RBF interpolation and POD. Audouze et al. (2013) proposed a non-intrusive reduced order modelling approach for nonlinear parameterised time-dependent PDEs based on a two-level POD method. This method is verified and validated using Burgers equation and convection-diffusion-reaction problems (Audouze et al., 2009, 2013). Xiao et al. (2015a) presented three non-intrusive reduced order methods for Navier-Stokes equations using hypersurfaces representation. The hypersurfaces are established by POD and RBF interpolation, Smolyak sparse grid and Taylor series expansion method (Xiao et al., 2015a, 2015b). In addition, the hypersurface method based on NIROM has been successfully applied to fluid-structure interaction problems (Xiao et al., 2016, 2017a) and free surface flow problems (Xiao et al., 2017b)

Recently, reduced order methods (e.g., POD, POD/DEIM, trajectory piecewise linearisation and bilinear approximation techniques) have been applied to reservoir modelling (Heijn et al., 2004; Cardoso, 2009; Cardoso et al., 2009; Chaturantabut and Sorensen, 2011; Yang et al., 2015; Yoon et al., 2014; Ghasemi et al., 2014; Klie et al., 2013). Heijn et al. (2004), Cardoso (2009) and Cardoso et al. (2009) first developed POD ROMs for reservoir simulation. Chaturantabut and Sorensen (2011), Yang et al. (2015) and Yoon et al. (2014) further introduced DEIM into model reduction for nonlinear flows (Chaturantabut and Sorensen, 2011). Again, these reduced order methods are intrusive and equations/codes dependent. On the other hand, there are very few studies in non-intrusive reduced order modelling in porous media flows and 3D fluvial channel 
problems. Klie et al. (2013) first proposed a non-intrusive model reduction approach based on a three-layer neural network combined with POD and DEIM to predict the production of oil and gas reservoirs, where the RBF neural network is used for learning about the input and output relationships. In this work, we used RBF as an interpolation method for constructing the time-dependent POD ROM.

In this work, we extend the hypersurfaces based NIROM (Xiao et al., 2015a) to 3D reservoir modelling, and applied to a fluvial channel problem. The novelty of this work lies in the hypersurfaces representation of 3D reservoir modelling on a reduced space under the framework of the Imperial College Finite Element Reservoir Simulator (IC-FERST). In this approach, solutions to the full fidelity 3D reservoir model are recorded using the snapshot methods, and from these snapshots POD bases are generated that optimally represent the $3 \mathrm{D}$ reservoir simulation. The RBF interpolation method is then used to form a set of hypersurfaces (interpolation functions) that approximate the time dependent ROM. After obtaining hypersurfaces, the solution of ROM at the current time level can be calculated by inputting POD coefficients of earlier time levels into the hypersurfaces. The capabilities of results from the new NIROM have been assessed by two 3D reservoir simulation test cases.

The structure of the article is as follows: Section 2 presents the governing equations of the 3D reservoir model; Section 3 presents the reduced order modelling method using hypersurfaces representation; Section 4 illustrates the methodology derived by means of two numerical examples. The illustration consists of two test problems where a horizontally layered porous media test case and a 3D fluvial channel case are resolved. Finally in Section 5, the conclusion is presented.

\section{Governing equations of 3D reservoir modelling}

The governing equations used in the underlying 3D reservoir model are given in this section. The Darcy's law for immiscible multiphase flow in porous media has the form:

$$
\mathbf{q}_{\alpha}=-\frac{\mathcal{K}_{r \alpha}}{\mu_{\alpha}} \mathbf{K}\left(\nabla p_{\alpha}-\mathbf{s}_{u \alpha}\right)
$$

where $\mathbf{q}_{\alpha}$ is the $\alpha^{\text {th }}$ phase Darcy velocity. The $\mathcal{K}_{r \alpha}$ is the relative permeability of the $\alpha^{\text {th }}$ phase, and it is a function that is denoted by $\mathcal{K}_{r \alpha}\left(S_{\alpha}\right)$ corresponding to the phase saturation variable $S_{\alpha} \cdot p_{\alpha}$ is the pressure of the $\alpha^{\text {th }}$ phase, which may include capillary pressure. $\mathbf{K}$ is the absolute permeability tensor of the porous medium. $\mu_{\alpha}$ and $\mathbf{s}_{u \alpha}$ are the phase dynamic viscosity and source term respectively, which may include gravity.

A saturation-weighted Darcy velocity is introduced into the equation (1) and defined as

$$
\mathbf{v}_{\alpha}=\frac{\mathbf{s}_{\alpha}}{S_{\alpha}}
$$

then equation (1) can be rewritten as follows:

$$
u_{\alpha}=\underset{=\alpha}{\sigma} \mathbf{v}_{\alpha}=-\nabla p_{\alpha}+\mathbf{s}_{u \alpha}, \underset{=\alpha}{\sigma}=\mu_{\alpha} S_{\alpha}\left(\mathcal{K}_{r \alpha} \mathbf{K}\right)^{-1}
$$


where $\mathbf{u}_{\alpha}$ denotes the force per unit volume, which is defined as $\underset{=}{\sigma} \mathbf{v}_{\alpha}$ and used as a prognostic variable in this approach. The $\underset{=\alpha}{\sigma}$ represents the implicit linearisation of the viscous frictional forces.

The saturation equation can be written as:

$$
\phi \frac{\partial S_{\alpha}}{\partial t}+\nabla \cdot\left(\mathbf{v}_{\alpha} S_{\alpha}\right)=S_{c t y, \alpha}
$$

where $\phi$ denotes the porosity. The $t$ is time and $s_{c t y, \alpha}$ is a source term of the $\alpha^{\text {th }}$ phase.

Finally, equation (4) is bounded by the constraint:

$$
\sum_{\alpha=1}^{N_{\alpha}} S_{\alpha}=1
$$

where $N_{\alpha}$ denotes the number of phases.

\subsection{Discretisation of the governing equations}

The discretisation of the above equations (1)-(5) at time level $n$ can be written in a general form:

$$
\mathbf{A}_{v}^{n} \mathbf{v}^{n}=\mathbf{s}_{v}^{n}, \quad \mathbf{A}_{p}^{n} \mathbf{p}^{n}=\mathbf{s}_{p}^{n}, \quad \mathbf{A}_{S}^{n} \mathbf{S}^{n}=\mathbf{s}_{S}^{n}
$$

where $\mathbf{v}^{n}=\left(\mathbf{v}_{1}^{n}, \ldots, \mathbf{v}_{\alpha}^{n}, \ldots, \mathbf{v}_{N_{\alpha}}^{n}\right)^{T}, \quad \mathbf{p}^{n}=\left(\mathbf{p}_{1}^{n}, \ldots, \mathbf{p}_{\alpha}^{n}, \ldots, \mathbf{p}_{N_{\alpha}}^{n}\right)^{T}$ and $\mathbf{S}^{n}=\left(\mathbf{S}_{1}^{n}, \ldots, \mathbf{S}_{\alpha}^{n}, \ldots\right.$, $\left.\mathbf{S}_{N_{\alpha}}^{n}\right)^{T} . \quad \mathbf{v}_{\alpha}^{n}, \mathbf{p}_{\alpha}^{n}$ and $\mathbf{S}_{\alpha}^{n}$ are velocity, pressure and saturation vectors of phase $\alpha$, and $\mathbf{v}_{\alpha}^{n}=\left(v_{\alpha, 1}, \ldots, v_{\alpha, \mathcal{N}}\right)^{T}, \mathbf{p}_{\alpha}^{n}=\left(P_{\alpha, 1}, \ldots, P_{\alpha, \mathcal{N}}\right)^{T}, \mathbf{S}_{\alpha}^{n}=\left(S_{\alpha, 1}, \ldots, S_{\alpha, \mathcal{N}}\right)^{T}$, and $\mathcal{N}$ is the number of nodes.

\section{Model reduction for 3D reservoir modelling}

In this section, the process of deriving a non-intrusive ROM (NIROM) for 3D reservoir model is described.

\subsection{Proper orthogonal decomposition formulation}

POD is a technique used to find a set of optimal basis functions from the snapshots of solutions obtained from the original model. The optimal POD basis functions are then used to formulate a reduced dynamical system that contains the main features of the flow. Due to the optimality of convergence in terms of kinetic energy of the POD basis functions, dominant components of a large dimensional process can be captured with only a small number of bases e.g., 10-100.

In this work, we find a set of basis functions for each phase $(\alpha)$ of the variables: velocity $\mathbf{v}_{\alpha}$, pressure $\mathbf{p}_{\alpha}$ and saturation $\mathbf{S}_{\alpha}$. At time level $n$, those variables can be expressed: 


$$
\mathbf{v}_{\alpha}^{n}=\overline{\mathbf{v}_{\alpha}}+\Phi_{\alpha, v}^{T} \mathbf{v}_{r, \alpha}^{n}, \mathbf{p}_{\alpha}^{n}=\overline{\mathbf{p}_{\alpha}}+\Phi_{\alpha, p}^{T} \mathbf{p}_{r, \alpha}^{n}, \mathbf{S}_{\alpha}^{n}=\overline{\mathbf{S}_{\alpha}}+\Phi_{\alpha, S}^{T} \mathbf{S}_{r, \alpha}^{n}, \alpha \in\left\{1,2, \ldots, N_{\alpha}\right\}
$$

where $\overline{\mathbf{v}_{\alpha}}, \overline{\mathbf{p}_{\alpha}}$ and $\overline{\mathbf{S}_{\alpha}}$ are the mean of the ensemble of snapshots for the variables $\mathbf{v}_{\alpha}^{n}, \mathbf{p}_{\alpha}^{n}$ and $\mathbf{S}_{\alpha}^{n}$ respectively, $\Phi_{\alpha, v}=\left(\Phi_{\alpha, v, 1}, \ldots, \Phi_{\alpha, v, M}\right), \Phi_{\alpha, p}=\left(\Phi_{\alpha, p, 1}, \ldots, \Phi_{\alpha, p, M}\right)$, $\Phi_{\alpha, v}=\left(\Phi_{\alpha, S, 1}, \ldots, \Phi_{\alpha, S, M}\right)$, are the POD bases for $\mathbf{v}_{\alpha}^{n}, \mathbf{p}_{\alpha}^{n}$ and $\mathbf{S}_{\alpha}^{n}$ respectively, which are extracted through truncated singular value decomposition, and $M$ is the number of POD bases used in the POD model. $\mathbf{v}_{r, \alpha}, \mathbf{p}_{r, \alpha}$ and $\mathbf{S}_{r, \alpha}$ denote phase $\alpha$ 's POD coefficients of velocity, pressure and saturation respectively. $\mathbf{v}_{r, \alpha}^{n}=\left(\mathbf{v}_{r, \alpha, 1}^{n}, \ldots, \mathbf{v}_{r, \alpha, j}^{n}, \ldots, \mathbf{v}_{r, \alpha M}^{n}\right)^{T}$, $\mathbf{p}_{r, \alpha}^{n}=\left(\mathbf{p}_{r, \alpha, 1}^{n}, \ldots, \mathbf{p}_{r, \alpha, j}^{n}, \ldots, \mathbf{p}_{r, \alpha M}^{n}\right)^{T}$ and $\mathbf{S}_{r, \alpha}^{n}=\left(\mathbf{S}_{r, \alpha, 1}^{n}, \ldots, \mathbf{S}_{r, \alpha, j}^{n}, \ldots, \mathbf{S}_{r, \alpha M}^{n}\right)^{T}$.

Projecting equation (6) onto the reduced space, yields:

$$
\Phi_{\alpha, v}^{T} \mathbf{A}_{\alpha, v}^{n} \Phi_{\alpha, v} \mathbf{v}_{\alpha}^{n}=\mathbf{s}_{v}^{n}, \quad \Phi_{\alpha, p}^{T} \mathbf{A}_{\alpha, p}^{n} \Phi_{\alpha, p} \mathbf{p}_{\alpha}^{n}=\mathbf{s}_{p}^{n}, \quad \Phi_{\alpha, S}^{T} \mathbf{A}_{\alpha, S}^{n} \Phi_{\alpha, S} \mathbf{S}_{\alpha}^{n}=\mathbf{s}_{S}^{n}
$$

The ROM for solving the POD coefficients $\mathbf{v}_{r, \alpha, j}^{n}, \mathbf{p}_{r, \alpha, j}^{n}$ and $\mathbf{S}_{r, \alpha, j}^{n}$ (where $j \in\{1,2, \ldots$, $\left.M\}, \alpha \in\left\{1,2, \ldots, N_{\alpha}\right\}\right)$ at time level $n$ can be written in the general form:

$$
\begin{aligned}
& \mathbf{v}_{r, \alpha, j}^{n}=f_{\alpha, v, j}\left(\mathbf{v}_{r, 1}^{n-1}, \mathbf{p}_{r, 1}^{n-1}, \mathbf{S}_{r, 1}^{n-1}, \ldots, \mathbf{v}_{r, \alpha}^{n-1}, \mathbf{p}_{r, \alpha}^{n-1}, \mathbf{S}_{r, \alpha}^{n-1}, \ldots, \mathbf{v}_{r, N_{\alpha}}^{n-1}, \mathbf{p}_{r, N_{\alpha}}^{n-1}, \mathbf{S}_{r, N_{\alpha}}^{n-1}\right), \\
& \mathbf{p}_{r, \alpha, j}^{n}=f_{\alpha, v, j}\left(\mathbf{v}_{r, 1}^{n-1}, \mathbf{p}_{r, 1}^{n-1}, \mathbf{S}_{r, 1}^{n-1}, \ldots, \mathbf{v}_{r, \alpha}^{n-1}, \mathbf{p}_{r, \alpha}^{n-1}, \mathbf{S}_{r, \alpha}^{n-1}, \ldots, \mathbf{v}_{r, N_{\alpha}}^{n-1}, \mathbf{p}_{r, N_{\alpha}}^{n-1}, \mathbf{S}_{r, N_{\alpha}}^{n-1}\right), \\
& \mathbf{S}_{r, \alpha, j}^{n}=f_{\alpha, \nu, j}\left(\mathbf{v}_{r, 1}^{n-1}, \mathbf{p}_{r, 1}^{n-1}, \mathbf{S}_{r, 1}^{n-1}, \ldots, \mathbf{v}_{r, \alpha}^{n-1}, \mathbf{p}_{r, \alpha}^{n-1}, \mathbf{S}_{r, \alpha}^{n-1}, \ldots, \mathbf{v}_{r, N_{\alpha}}^{n-1}, \mathbf{p}_{r, N_{\alpha}}^{n-1}, \mathbf{S}_{r, N_{\alpha}}^{n-1}\right),
\end{aligned}
$$

subject to the initial condition

$$
\mathbf{v}_{r, \alpha, j}^{0}=\left(\left(\mathbf{v}_{\alpha, j}^{0}-\overline{\mathbf{v}_{\alpha}}\right), \Phi_{\alpha, j}\right), \mathbf{p}_{r, \alpha, j}^{0}=\left(\left(\mathbf{p}_{\alpha, j}^{0}-\overline{\mathbf{p}_{\alpha}}\right), \Phi_{\alpha, j}\right), \mathbf{S}_{r, \alpha, j}^{0}=\left(\left(\mathbf{S}_{\alpha, j}^{0}-\overline{\mathbf{S}_{\alpha}}\right), \Phi_{\alpha, j}\right),
$$

where $\left(\mathbf{v}_{r, 1}^{n-1}, \mathbf{p}_{r, 1}^{n-1}, \mathbf{S}_{r, 1}^{n-1}, \ldots, \mathbf{v}_{r, \alpha}^{n-1}, \mathbf{p}_{r, \alpha}^{n-1}, \mathbf{S}_{r, \alpha}^{n-1}, \ldots, \mathbf{v}_{r, N_{\alpha}}^{n-1}, \mathbf{p}_{r, N_{\alpha}}^{n-1}, \mathbf{S}_{r, N_{\alpha}}^{n-1}\right)$ denotes a complete set of POD coefficients for solution fields velocities $\mathbf{v}$, pressures $\mathbf{p}$ and saturations $\mathbf{S}$ of all phases at time step $n-1\left(n \in\left\{1,2, \ldots, N_{t}\right\}\right), N t$ is the number of time levels in the computational simulation.

\subsection{Hypersurfaces based on RBF interpolation}

The RBF is an efficient method for interpolation problems. In this work, the RBF interpolation method is used to construct a set of hypersurfaces for reduced order $3 \mathrm{D}$ reservoir model. The theory of RBF interpolation is briefly reviewed in this section. The $\mathrm{RBF}$ interpolation method constructs an approximation function using the form of,

$$
f(\mathbf{x})=\sum_{i=1}^{N} w_{i} \phi\left(\left\|\mathbf{x}-\mathbf{x}_{i}\right\|\right)
$$

where the interpolation function (hypersurface) $f(\mathbf{x})$ is represented as a linear combination of $N$ RBFs $(\phi) . N$ denotes the number of RBFs. Each RBF is associated with a different centre $\mathbf{x}_{i}$, and weighted by a coefficient $w_{i} . \mathbf{x}$ denotes a data point in multidimensional space and it consists of a complete set of POD coefficients for solution fields of all phases such as velocity and pressure, saturation. In this work 
$\mathbf{x}=\left(\mathbf{v}_{r, 1}^{n-1}, \mathbf{p}_{r, 1}^{n-1}, \mathbf{S}_{r, 1}^{n-1}, \ldots, \mathbf{v}_{r, \alpha}^{n-1}, \mathbf{p}_{r, \alpha}^{n-1}, \mathbf{S}_{r, \alpha}^{n-1}, \ldots, \mathbf{v}_{r, N_{\alpha}}^{n-1}, \mathbf{p}_{r, N_{\alpha}}^{n-1}, \mathbf{S}_{r, N_{\alpha}}^{n-1}\right) . \quad\left\|\mathbf{x}-\mathbf{x}_{i}\right\| \quad$ is $\quad$ a scalar distance defined by the $L_{2}$ norm.

The weight coefficients $w_{i}$ are determined by ensuring that the interpolation function values $f(\mathbf{x})$ will match the given data y exactly. This is achieved by enforcing $f(\mathbf{x})=y$, which produces a linear equation

$$
B w=y,
$$

where

$$
\begin{aligned}
B & =\left[\begin{array}{cccc}
\phi\left(\left\|\mathbf{x}_{1}-\mathbf{x}_{1}\right\|_{2}\right) & \phi\left(\left\|\mathbf{x}_{1}-\mathbf{x}_{2}\right\|_{2}\right) & \cdots & \phi\left(\left\|\mathbf{x}_{1}-\mathbf{x}_{n}\right\|_{2}\right) \\
\phi\left(\left\|\mathbf{x}_{2}-\mathbf{x}_{1}\right\|_{2}\right) & \phi\left(\left\|\mathbf{x}_{2}-\mathbf{x}_{2}\right\|_{2}\right) & \cdots & \phi\left(\left\|\mathbf{x}_{2}-\mathbf{x}_{n}\right\|_{2}\right) \\
\vdots & \vdots & \\
\phi\left(\left\|\mathbf{x}_{n}-\mathbf{x}_{1}\right\|_{2}\right) & \phi\left(\left\|\mathbf{x}_{n}-\mathbf{x}_{2}\right\|_{2}\right) & \cdots & \phi\left(\left\|\mathbf{x}_{n}-\mathbf{x}_{n}\right\|_{2}\right)
\end{array}\right] \\
w & =\left[w_{1}, w_{2}, \ldots, w_{n}\right]^{T}, y=\left[y_{1}, y_{2}, \ldots, y_{n}\right]^{T}
\end{aligned}
$$

The weight coefficients $w_{j}$ are then determined by solving the linear system (12) $A w=y$. How to define an appropriate $\operatorname{RBF} \phi$ is also important. The most well-known choices for $\phi$ are Gaussian $\left(\phi(r)=e^{-(r / \sigma)^{2}}\right)$, multi-quadratic inverse $\left(\phi(r)=\sqrt{r^{2}+\sigma^{2}}\right)$, multi-quadratic $\left(\phi(r)=\frac{1}{\sqrt{r^{2}+\sigma^{2}}}\right)$, inverse quadratic $\phi(r)=\frac{1}{r^{2}+\sigma^{2}}$ and thin plate spline $\left(\phi(r)=r^{2} \log r\right) . r \geq 0$ is a radius and $\sigma>0$ is a shape parameter.

\subsection{Hypersurfaces representing the reduced system of the 3D reservoir model}

In this section the procedure of forming a set of hypersurfaces for 3D reservoir model in a reduced space is described. This is achieved by using Gaussian RBF interpolation method to construct the ROM in (9). By applying the RBF method, a set of hypersurfaces $f_{\alpha, v, j}^{n}, f_{\alpha, p, j}^{n}$ and $f_{\alpha, S, j}^{n}$ for each POD coefficient $v_{r, \alpha, j}^{n}, p_{r, \alpha, j}^{n}$ and $S_{r, \alpha, j}^{n}$ (where $j \in\{1,2$, $\left.\ldots, M\}, \alpha \in\left\{1,2, \ldots, N_{\alpha}\right\}\right)$ be approximately represented by the interpolation functions below:

$$
\begin{aligned}
& \mathbf{v}_{r, \alpha, j}^{n}=f_{\alpha, v, j}\left(\mathbf{v}_{r, 1}^{n-1}, \mathbf{p}_{r, 1}^{n-1}, \mathbf{S}_{r, 1}^{n-1}, \ldots, \mathbf{v}_{r, \alpha}^{n-1}, \mathbf{p}_{r, \alpha}^{n-1}, \mathbf{S}_{r, \alpha}^{n-1}, \ldots, \mathbf{v}_{r, N_{\alpha}}^{n-1}, \mathbf{p}_{r, N_{\alpha}}^{n-1}, \mathbf{S}_{r, N_{\alpha}}^{n-1}\right)=\sum_{i=1}^{N} w_{\alpha, v, i, j} * \phi\left(\mathbf{r}_{i}\right), \\
& \mathbf{p}_{r, \alpha, j}^{n}=f_{\alpha, v, j}\left(\mathbf{v}_{r, 1}^{n-1}, \mathbf{p}_{r, 1}^{n-1}, \mathbf{S}_{r, 1}^{n-1}, \ldots, \mathbf{v}_{r, \alpha}^{n-1}, \mathbf{p}_{r, \alpha}^{n-1}, \mathbf{S}_{r, \alpha}^{n-1}, \ldots, \mathbf{v}_{r, N_{\alpha}}^{n-1}, \mathbf{p}_{r, N_{\alpha}}^{n-1}, \mathbf{S}_{r, N_{\alpha}}^{n-1}\right)=\sum_{i=1}^{N} w_{\alpha, p, i, j} * \phi\left(\mathbf{r}_{i}\right), \\
& \mathbf{S}_{r, \alpha, j}^{n}=f_{\alpha, v, j}\left(\mathbf{v}_{r, 1}^{n-1}, \mathbf{p}_{r, 1}^{n-1}, \mathbf{S}_{r, 1}^{n-1}, \ldots, \mathbf{v}_{r, \alpha}^{n-1}, \mathbf{p}_{r, \alpha}^{n-1}, \mathbf{S}_{r, \alpha}^{n-1}, \ldots, \mathbf{v}_{r, N_{\alpha}}^{n-1}, \mathbf{p}_{r, N_{\alpha}}^{n-1}, \mathbf{S}_{r, N_{\alpha}}^{n-1}\right), \sum_{i=1}^{N} w_{\alpha, S, i, j} * \phi\left(\mathbf{r}_{i}\right),
\end{aligned}
$$

where $\phi\left(r_{i}\right)$ is the RBF whose values depend on the distance from a collection of centre points, $\left(\hat{\mathbf{v}}_{r, 1}^{n-1}, \hat{\mathbf{p}}_{r, 1}^{n-1}, \hat{\mathbf{S}}_{r, 1}^{n-1}, \ldots, \hat{\mathbf{v}}_{r, \alpha}^{n-1}, \hat{\mathbf{p}}_{r, \alpha}^{n-1}, \hat{\mathbf{S}}_{r, \alpha}^{n-1}, \ldots, \hat{\mathbf{v}}_{r, N_{\alpha}}^{n-1}, \hat{\mathbf{p}}_{r, N_{\alpha}}^{n-1}, \hat{\mathbf{S}}_{r, N_{\alpha}}^{n-1}\right)$ (where $j \in\{1,2, \ldots$, 
$\left.M\}, \alpha \in\left\{1,2, \ldots, N_{\alpha}\right\}\right)$ and weighted by $w_{\alpha, v, i}, w_{\alpha, p, i}$, and $w_{\alpha, S, i}$. In this work, the Gaussian $\mathrm{RBF}$ is chosen:

$$
\phi\left(\mathbf{r}_{i}\right)=e^{-\left(\mathbf{r}_{i} / \sigma\right)^{2}}=e^{-\left(\left\|\left(\mathbf{v}_{r}^{n-1}, \mathbf{p}_{r}^{n-1}, \mathbf{S}_{r}^{n-1}\right)-\left(\hat{\mathbf{v}}_{r, i}, \hat{\mathbf{p}}_{r, i}, \hat{\mathbf{s}}_{r, i}\right)\right\| / \sigma^{2}\right)}
$$

where $\mathbf{v}_{r}$ denotes the POD coefficients of velocity including all phases, $\mathbf{v}_{r}=\left(\mathbf{v}_{r, 1}, \ldots, \mathbf{v}_{r, \alpha}, \ldots, \mathbf{v}_{r, N_{\alpha}}\right)$. This applies to pressure vector $\mathbf{p}_{r}^{n-1}$ and saturation vector $\mathbf{S}_{r}^{n-1} \cdot r_{i}$ is a radius or the distance defined by the $L_{2}$ norm, $\sigma>0$ is a shape parameter. $\left(\hat{\mathbf{v}}_{r, i}, \hat{\mathbf{p}}_{r, i}, \hat{\mathbf{S}}_{r, i}\right)$ denotes a centre point. The weighting coefficients $w_{\alpha, v, i}, w_{\alpha, p, i}$, and $w_{\alpha, S, I}$ are determined so as to ensure that the interpolation function values at the collection data point $\left(\mathbf{v}_{r, k}, \mathbf{p}_{r, k}, \mathbf{S}_{r, k}\right)$ match the given data $f_{\alpha, v, k}, f_{\alpha, p, k}$ and $f_{\alpha, S, k}$. This can be expressed by,

$$
B \mathbf{w}_{\alpha, v, i, j}=\mathbf{f}_{\alpha, v, i, j}, B \mathbf{w}_{\alpha, p, i, j}=\mathbf{f}_{\alpha, p, i, j}, B \mathbf{w}_{\alpha, S, i, j}=\mathbf{f}_{\alpha, S, i, j}, i \in\{1,2, \ldots, N\},
$$

where

- $\quad \mathbf{w}_{\alpha, v, i}=\left(w_{\alpha, v, i, k}\right)_{k=1, \ldots, N}^{T}, \mathbf{w}_{\alpha, p, i}=\left(w_{\alpha, p, i, k}\right)_{k=1, \ldots, N}^{T}$ and $\mathbf{w}_{\alpha, S, i}=\left(w_{\alpha, S, i, k}\right)_{k=1, \ldots, N}^{T}$

- $\quad \mathbf{f}_{\alpha, v, i}=\left(w_{\alpha, v, i, k}\right)_{k=1, \ldots, N}^{T}, \mathbf{f}_{\alpha, p, i}=\left(w_{\alpha, p, i, k}\right)_{k=1, \ldots, N}^{T}$ and $\mathbf{f}_{\alpha, S, i}=\left(w_{\alpha, S, i, k}\right)_{k=1, \ldots, N}^{T}$

- $B$ is the interpolation matrix of elements $B_{k, l}=\phi\left(\mathbf{r}_{k, l}\right)$

- $k, l \in\{1,2, \ldots, N\}, N$ is the number of data points.

The weighting coefficients $\mathbf{w}_{\alpha, v, i, j}, \mathbf{w}_{\alpha, p, i, j}$ and $\mathbf{w}_{\alpha, S, i, j}$ are then determined by solving the linear system (17).

\subsection{Summary of constructing the NIROM for the 3D reservoir model}

In this section, the algorithm of constructing the hypersurfaces and obtaining results using the NIROM is summarised, which includes the offline process and online process. The offline process consists of obtaining the basis functions and constructing the hypersurfaces.

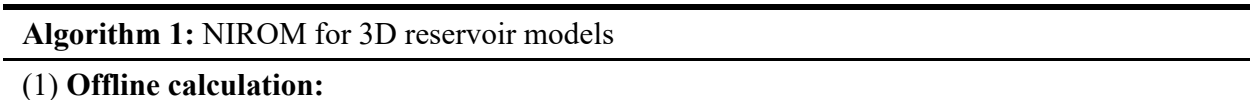

(a) Obtaining the POD bases for 3D reservoir model

(i) Generate the snapshots at time level $n=1, \ldots, N_{t}$ by solving the $3 \mathrm{D}$ reservoir model (6);

(ii) Construct the POD bases $\Phi_{v}, \Phi_{p}$ and $\Phi_{S}$ using the SVD method;

(b) Construct a set of hypersurfaces

(i) Calculate the functional values $f_{v, i, k}, f_{p, i, k}$ and $f_{S, i, k}$ at the data point $\left(\mathbf{v}_{r, k}, \mathbf{p}_{r, k}, \mathbf{S}_{r, k}\right)$ through the solution from the full models, where $k \in\{1,2, \ldots, N\}$;

(ii) Find the weights $\mathbf{w}_{v, i}, \mathbf{w}_{p, i}$ and $\mathbf{w}_{S, i}$ by solving (17) such that the hypersurfaces $\mathbf{f}_{v, i}$, $\mathbf{f}_{p, i}$ and $\mathbf{f}_{S, i}$ pass through the data points;

(2) Online calculation: The hypersurfaces in (15) denotes a $3 \alpha \mathrm{M}$-dimensional hyper surface Once a set of interpolation functions $f_{\alpha, v, j}, f_{\alpha, p, j}$ and $f_{\alpha, S, j}$ are constructed, they are then used to estimate the $j^{\text {th }}$ POD coefficient of phase $(\alpha) v_{r, \alpha, j}^{n}, p_{r, \alpha, j}^{n}$ and $S_{r, \alpha, j}^{n}$ at time level $n$. 


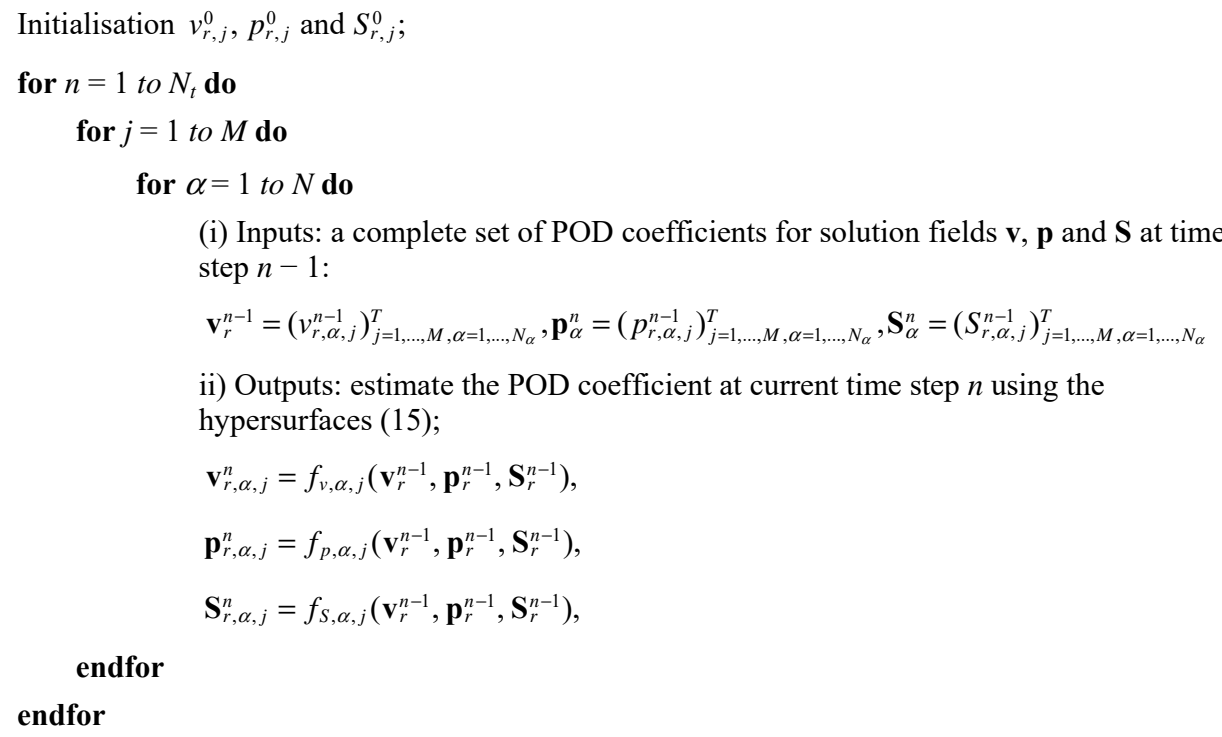

Obtain the solution of variables $\mathbf{v}_{\alpha}^{n}, \mathbf{p}_{\alpha}^{n}$ and $\mathbf{S}_{\alpha}^{n}$ in (4) by projecting $v_{r, \alpha, j}^{n}, p_{r, \alpha, j}^{n}$ and $S_{r, \alpha, j}^{n}$ onto the full space (see (7)).

$$
\mathbf{v}_{\alpha}^{n}=\overline{\mathbf{v}_{\alpha}}+\Phi_{v, \alpha}^{T} \mathbf{v}_{r, \alpha}^{n}, \mathbf{p}_{\alpha}^{n}=\overline{\mathbf{p}_{\alpha}}+\Phi_{p, \alpha}^{T} \mathbf{p}_{r, \alpha}^{n}, \mathbf{S}_{\alpha}^{n}=\overline{\mathbf{S}_{\alpha}}+\Phi_{S, \alpha}^{T} \mathbf{S}_{r, \alpha}^{n},
$$

endfor

\section{Numerical examples}

\subsection{Introduction of an unstructured mesh 3D reservoir model}

The NIROM has been implemented under the framework of an advanced 3D unstructured mesh reservoir model (IC-FERST). A novel control volume finite element method (CVFEM) is used to obtain the high-order fluxes on CV boundaries which are limited to yield bounded fields (e.g., positive saturations). This method is combined with a novel family of FE pairs, originally introduced for geophysical fluid dynamics applications. In particular, the $P 2 D G-P 1 D G$ element pair (quadratic discontinuous polynomial FE basis function for velocity $(P 2 D G)$ and linear discontinuous polynomial FE basis function for pressure, $P 1 D G)$, is used to accurately represent sharp saturation changes between heterogeneous domains, see Salinas et al. (2015) and Su et al. (2015).

The water-flooding is a widely known technique in oil and gas reservoir engineering. It increases the production from oil reservoirs through injecting water into the reservoir. As illustrated in Figure 1, the water is injected into the reservoir to increase the reservoir pressure; the oil is then displaced toward the production well. This phenomenon is also referred to the immiscible displacement in porous media. In this section, the capability of the NIROM developed for 3D unstructured mesh reservoir modelling has been numerically illustrated in a horizontally layered porous media case, and then further applied to a 3D complex fluvial channel case. 
Figure 1 Water-flooding technique for oil production (see online version for colours)

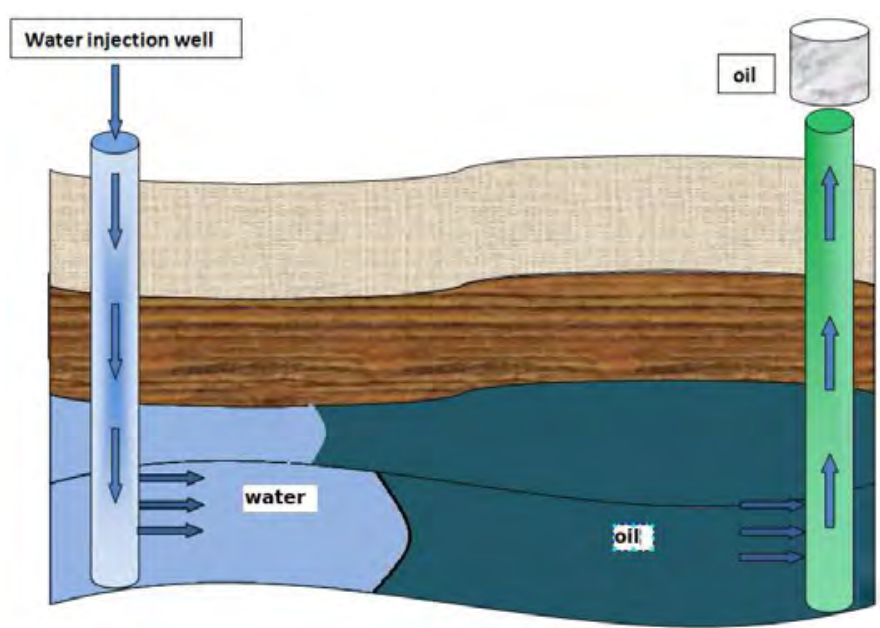

\subsection{Case 1: horizontally layered porous media case (simple test case)}

The first case for numerical illustration of the method proposed in this paper is a horizontally layered porous media case. This test case is dimensionless and for simplicity no gravity has been considered. This problem domain is consisting of a rectangle of non-dimensional size $1 \times 0.2$. The domain is divided into two identical areas with a permeability ratio of 4 (non-dimensional size) within the top half part and 1 (non-dimensional size) on the bottom half part. All the units in this paper are non-dimensional size. The outlet boundary has a dimensionless pressure of 0 (non-dimensional size), the whole domain is initially saturated with the non-wetting phase and the wetting phase at the irreducible saturation. The wetting phase is injected over the inlet boundary with a dimensionless velocity of 1 . The viscosity ratio of the phases is 1 . The Brooks-Corey model for the relative permeability, with an exponent of 2 and an end-point relative permeability ratio of 1 , is considered for both phases. The porosity is homogeneous and equal to 0.2 . The immobile fraction of the wetting phase is set to 0.2 and 0.3 for the non-wetting phase.

The problem was resolved with a mesh of 984 nodes during the simulation time period $[0,0.02]$. Fifty snapshots were taken from the pre-computed solution at regularly spaced time intervals $\Delta t=0.0002$ and from these POD bases are generated for the solution variables $v, p, S$.

The first 18 POD bases are presented in Figure 2. As shown in the figure, the first four POD bases capture most of flow features while the 5th-18th POD bases capture the details of small scale flow structures. Figure 3 shows the singular eigenvalues in order of decreasing magnitude. In general, the more POD bases and snapshots are chosen, the better the energy is represented. There is a trade-off between the accuracy and the CPU time. In this work, 18 POD bases with 50 snapshots are chosen resulting in $92 \%$ of 'energy' being captured.

Figure 4 shows the saturation solutions of the horizontally layered porous media problem at time instances $t=0.01$ and $t=0.02$, as calculated using the full and NIROMs. 
It can be seen that both model solutions are in good agreement with each other. The NIROM performs well in capturing the saturation shock-front.

Figure 2 Case 1: the figures show the first 18 POD bases functions of the horizontally layered porous media problem, (a) the 1st POD bases (b) the 2nd POD bases (c) the 3rd POD bases (d) the 4th POD bases (e) the 5th POD bases (f) the 8th POD bases (g) the 12th POD bases (h) the 18th POD bases (see online version for colours)

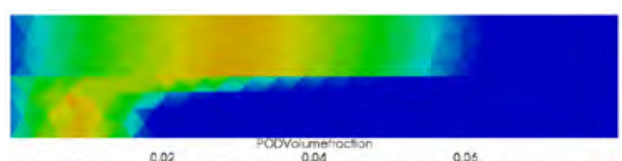

0.02

(a)

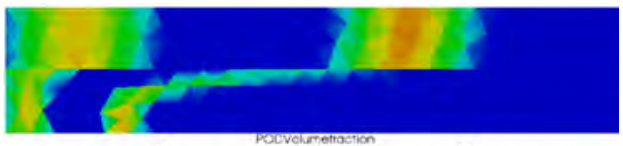

0.02

(c)

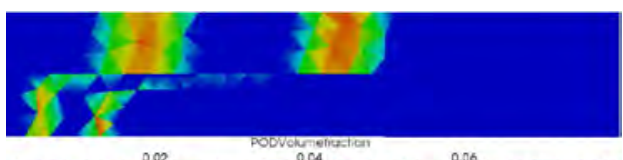

(e)

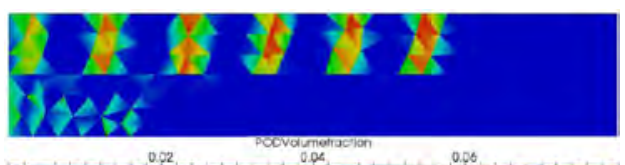

(g)

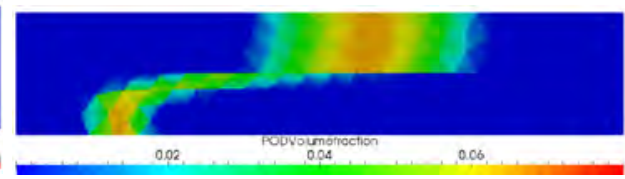

(b)

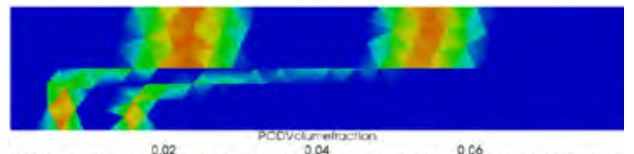

(d)

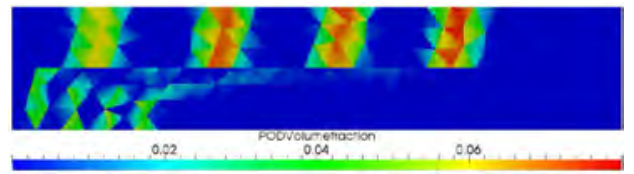

(f)

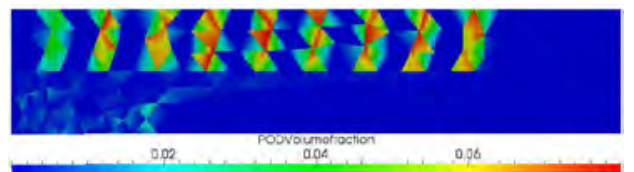

(h)

Figure 3 Case 1: the figure shows the singular eigenvalues in order of decreasing magnitude

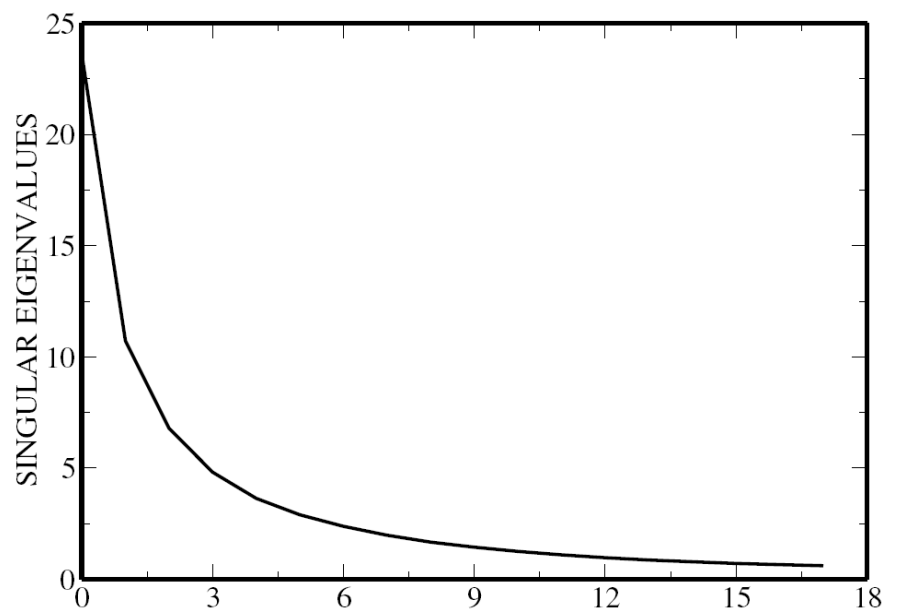


Figure 5 shows the saturation solution at a particular position $(0.026937,0.16246)$. It is noted the results from the NIROM using 6 and 12 POD bases become oscillatory after $t=10$. By increasing the number of POD bases from 6 to 18, the NIROM becomes stable and exhibits an overall good agreement with the full modelling.

The ability of the NIROM is further highlighted in Figure 6, which presents the saturation solution along a line parallel to the $\mathrm{x}$-axis. We can see the NIROM has a large error near the shock-front when using six POD bases. This can be significantly improved as the number of POD bases increases. Using 18 POD bases, the error of saturation solutions is decreased by $50 \%-97 \%$ in comparison to that using only six POD bases, and the shock-front is captured well.

Figure 4 Case 1: the figures show the saturation solutions of the horizontally layered porous media problem at time instances 0.01 and 0.02 , (a) full model, $\mathrm{t}=0.01$ (b) full model, $\mathrm{t}=0.02$ (c) NIROM 6 POD bases, $\mathrm{t}=0.01$ (d) NIROM 6 POD bases, $\mathrm{t}=0.02$

(e) NIROM 12 POD bases, $\mathrm{t}=0.01$ (f) NIROM 12 POD bases, $\mathrm{t}=0.02$

(g) NIROM 18 POD bases, $\mathrm{t}=0.01$ (h) NIROM 18 POD bases, $\mathrm{t}=0.02$ (see online version for colours)

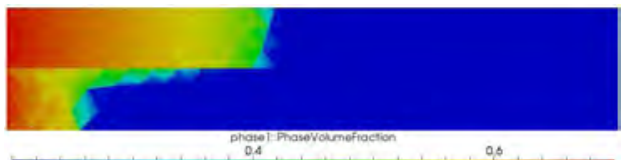

(a)

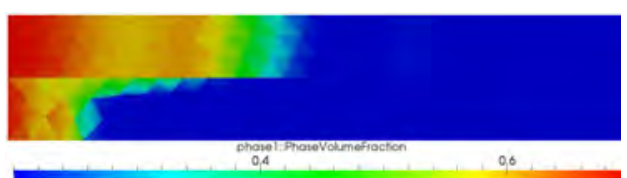

(c)

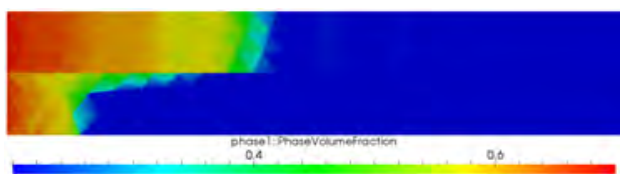

(e)

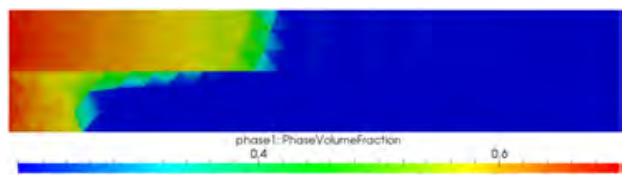

(g)

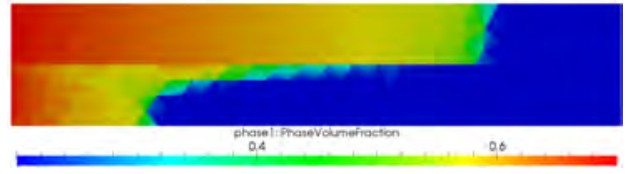

(b)

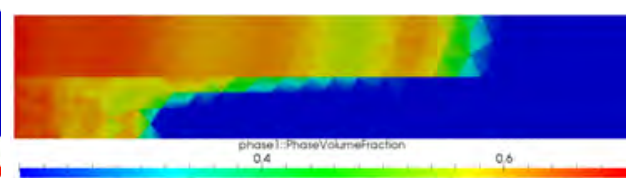

(d)

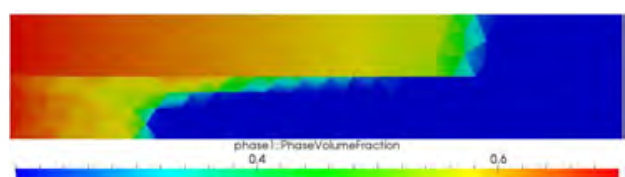

(f)

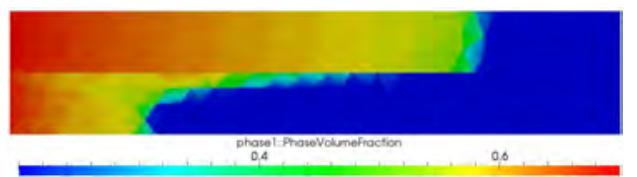

(h)

Notes: Where 6, 12 and 18 POD bases are chosen with 50 snapshots.

The permeability ratio on the top half part is 4 , and the bottom half part is 1 .

To further validate the quality of the NIROM, the corresponding error estimation of the POD ROM was carried out in this work. The accuracy of NIROM was assessed. The correlation coefficient of solutions between the full and NIROMs is computed for each 
time step, and is defined for given expected values $S_{\text {full }}^{n}$ and $S^{n}$ and standard deviations $\sigma_{S_{\text {full }}^{n}}$ and $\sigma_{S^{n}}$,

$$
\operatorname{corr}\left(S_{\text {full }}^{n}, S^{n}\right)^{n}=\frac{\operatorname{cov}\left(S_{\text {full }}^{n}, S^{n}\right)}{\sigma_{S_{\text {full }}^{n}} \sigma_{S^{n}}}=\frac{E\left[\left(S_{\text {full }}^{n}-\overline{S_{\text {full }}^{n}}\right)\left(S^{n}-\overline{S^{n}}\right)\right]}{\sigma_{S_{\text {full }}^{n}} \sigma_{S^{n}}}
$$

where $E$ denotes mathematical expectation, cov denotes covariance, $\sigma$ denotes standard deviation. $\overline{S_{\text {full }}^{n}}$ and $\overline{S^{n}}$ denote the mean of $S_{\text {full }}^{n}$ and $S^{n}$ respectively. The measured error is given by the root mean square error (RMSE) which is calculated for each time step $n$ by,

$$
R M S E^{n}=\sqrt{\frac{\sum_{i=1}^{N}\left(S_{\text {full }, i}^{n}-S_{i}^{n}\right)^{2}}{N}}
$$

In this expression $S_{\text {full,i }}^{n}$ and $S_{i}^{n}$ denote the full and NIROM solutions at the node $i$, respectively, and $N$ represents number of nodes on the full mesh. The RMSE and correlation coefficient of saturation solutions between the full and NIROMs are presented in Figure 7. With an increase in the number of POD bases, the RMSE in the saturation results decreases by about $50 \%$ while the correlation increases up to $98 \%$.

Figure 5 Case 1: the graph shows the solution saturations predicted by the full model and the NIROM at a position $(0.026937,0.16246)$ (see online version for colours)

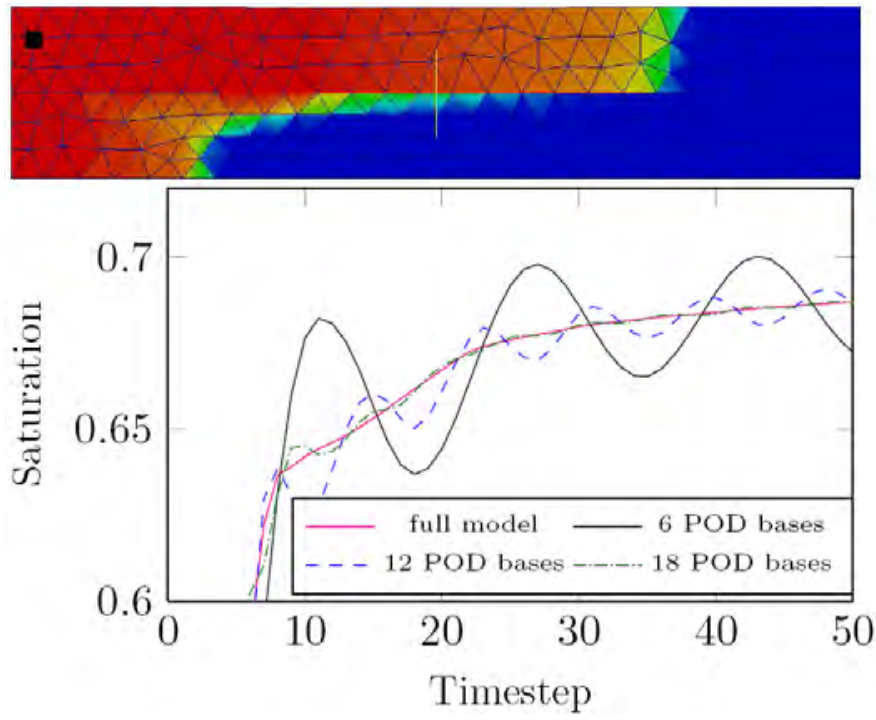

Note: Where 6, 12 and 18 POD bases are chosen with 50 snapshots. 
Figure 6 Case 1: saturation along lines parallel to the $\mathrm{x}$ axis, (a) the cross section locations (b) saturation at the cross section along the top line (c) saturation at the cross section along the bottom line (see online version for colours)

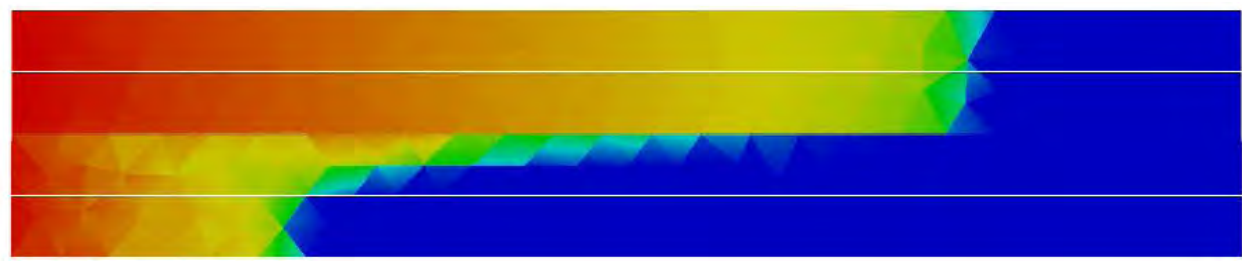

(a)

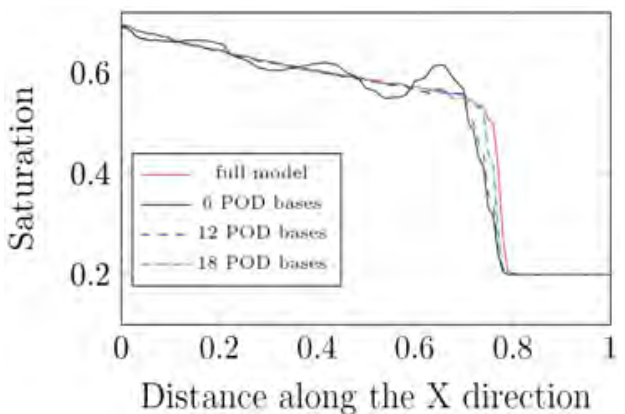

(b)

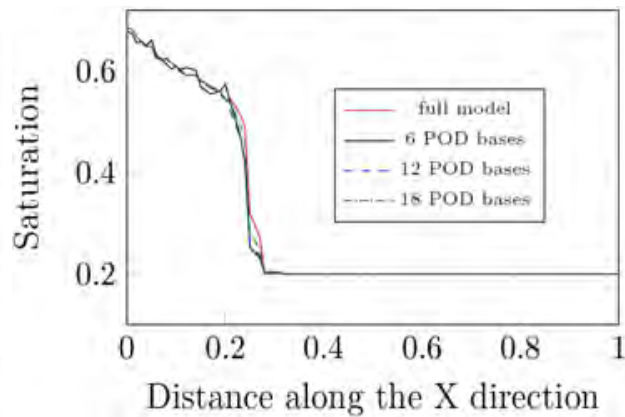

(c)

Figure 7 Case 1: the graph shows the RMSE and correlation coefficient of solutions between the full and NIROMs, (a) RMSE (b) correlation

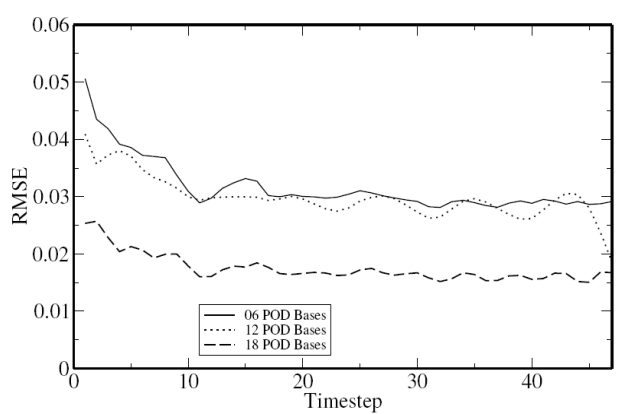

(a)

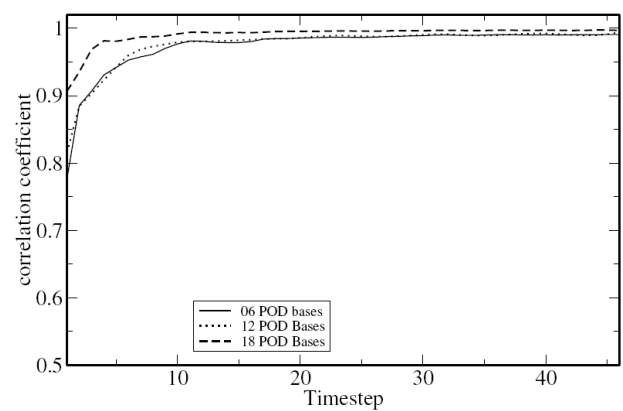

(b)

\subsection{Case 2: 3D fluvial channel case (complicated test case)}

The second case is a 3D fluvial channel case. There is a set of channels in the computational domain which is composed of 31,776 nodes, see Figure 8 . The domain is initially filled with immovable water and movable oil and the saturations of oil and water are $S_{\text {oil }}=0.8$ and $S_{\text {water }}=0.2$ respectively. The water is then injected into the computational domain from the right side at constant pressure. The flow passes through the channels from right side to the left side. All other sides are treated as barriers to flows except for the inlet side (right boundary in the computational domain) with a pressure of 
$55 \times 106$ and outlet side (left boundary) with a pressure of $2 \times 106$. The homogeneous porosity is set to be $\phi=0.2$ and the saturations of the residual oil and irreducible water are set to be 0.2 . The viscosities of the residual oil and irreducible water are set to be 0.004 and 0.001 respectively. The simulation was run during the simulation period $[0,1,000$ days $]$ with a time step size of 10 days. 100 snapshots of solutions were taken at regularly spaced time intervals $\Delta t=10$ days for each solution variable.

Figure 8 Case 2: the figures show the water channel profile, (a) water channel profile (top to bottom) (b) water channel profile (bottom to top) (see online version for colours)

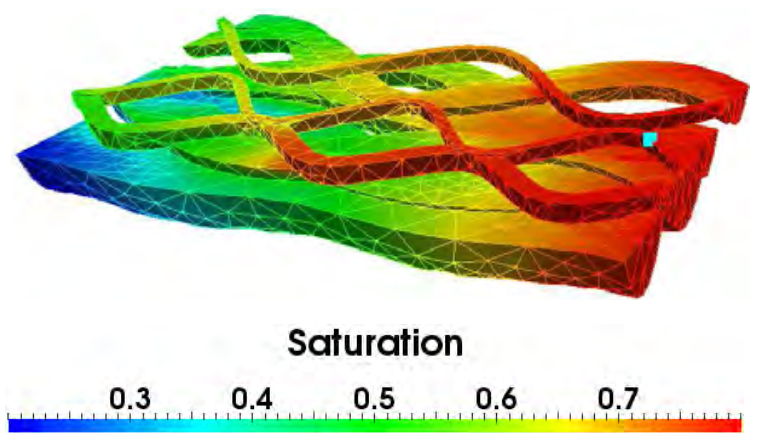

(a)

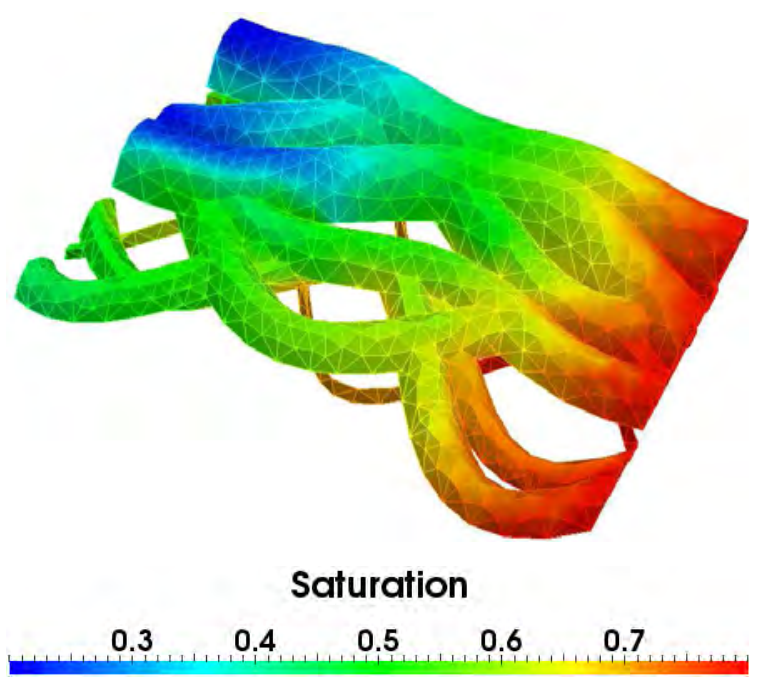

(b)

Figure 10 shows the first 36 leading POD bases functions of saturation. As shown in the figure, these leading POD bases capture the dominant characteristics of solutions. The POD bases corresponding to small eigenvalues, for example, the 30th and 36th POD bases contain small scale flow features. Figure 9 shows the singular eigenvalues in order of decreasing magnitude. As shown in the figure, the first six POD bases almost captured all of the total energy (96.83\%). 
Figure 9 Case 2: the figure shows the singular eigenvalues in order of decreasing magnitude

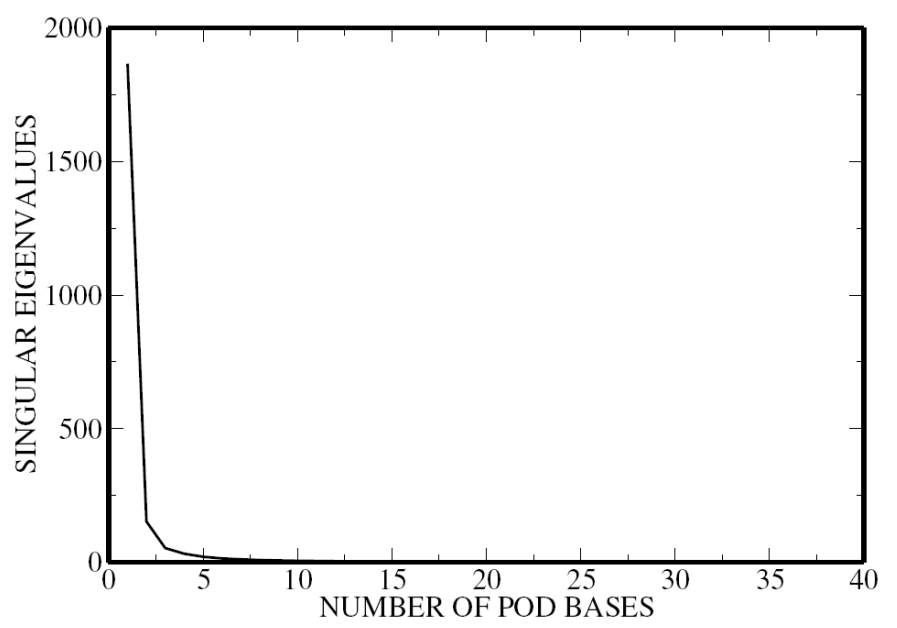

Figure 10 Case 2: the figures show the first 36 POD bases functions of the 3D fluvial channel problem, (a) the 1st POD bases (b) the 2nd POD bases (c) the 12th POD bases (d) the 24th POD bases (e) the 30th POD bases (f) the 36th POD bases (see online version for colours)

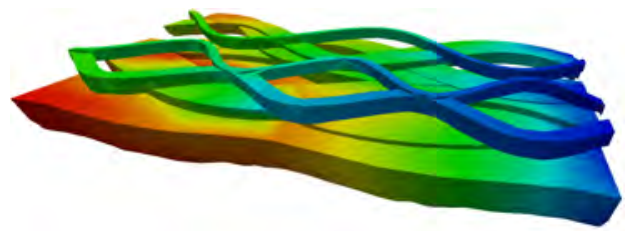

(a)

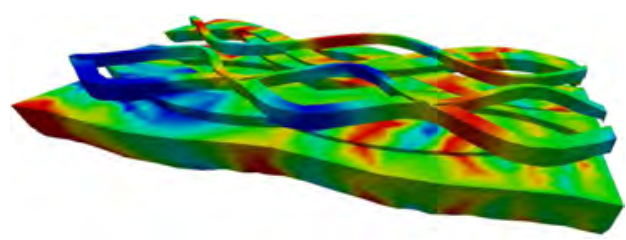

(c)

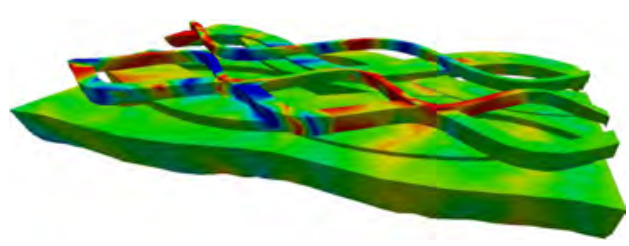

(e)

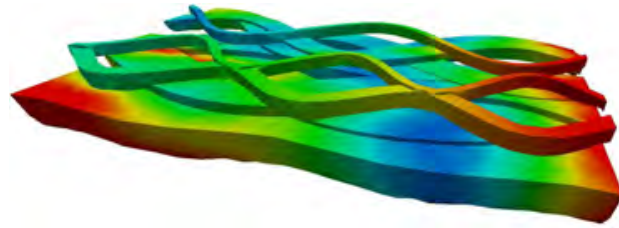

(b)

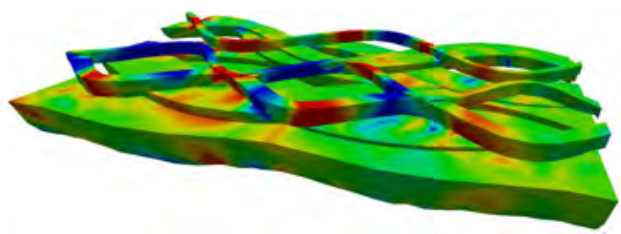

(d)

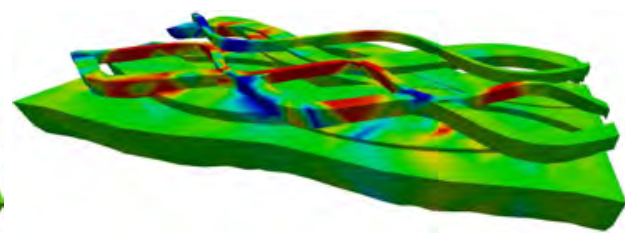

(f)

$\begin{array}{lllllll}-0.0075 & -0.005 & -0.0025 & \mathbf{0} & 0.0025 & 0.005 & 0.0075\end{array}$

$0.0075-0.005 \quad 0.0025 \quad \mathbf{0}$ $0.0025 \quad 0.005 \quad 0.0075$ 
Figure 11 Case 2: the figures show the saturation of full model and NIROM of the 3D fluvial channel problem at time instances 500 days and 800 days seconds using 6 and 36 POD bases, (a) full model, $\mathrm{t}=500$ days (b) full model, $\mathrm{t}=800$ days (c) NIROM (6 POD bases), $\mathrm{t}=500$ days (d) NIROM (6 POD bases), $\mathrm{t}=800$ days (e) NIROM (36 POD bases), $\mathrm{t}=500$ days (f) NIROM (36 POD bases), $\mathrm{t}=800$ days (see online version for colours)

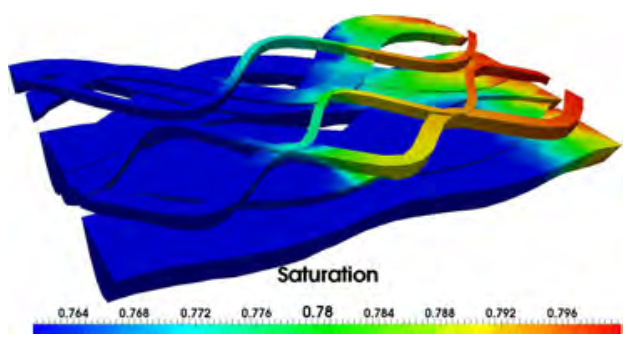

(a)

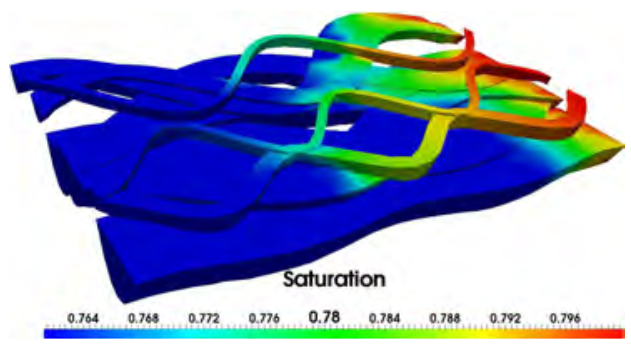

(c)

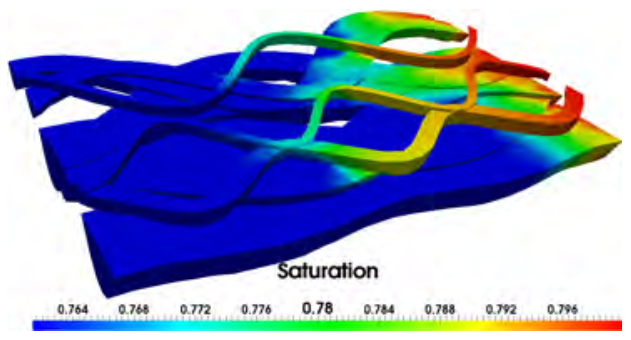

(f)

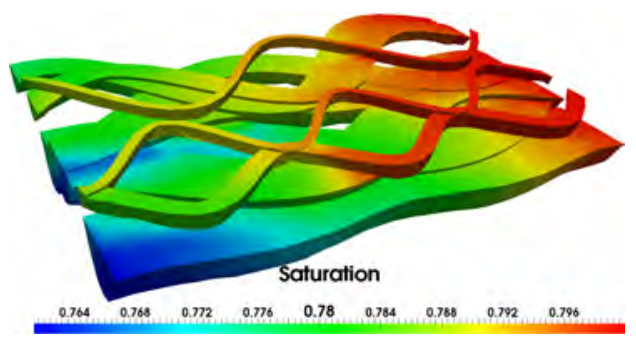

(b)

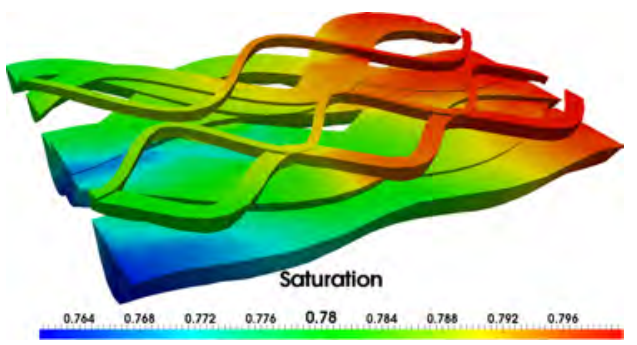

(d)

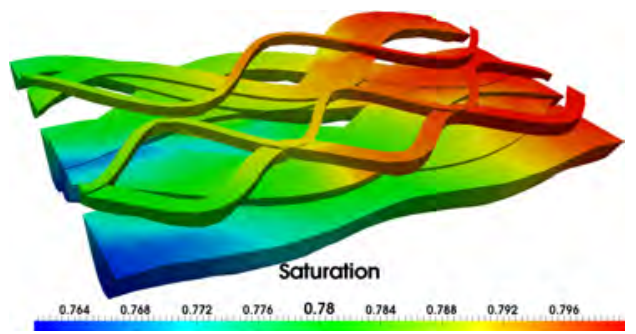

(g)

Figure 12 Case 2: the figures show above show the values of saturation at a particular point in the mesh-see figure (a), (a) location of the point (b) saturation values of the point (see online version for colours)

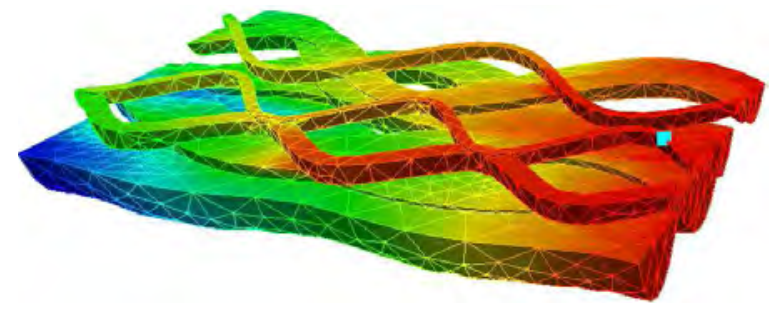

(a) 
Figure 12 Case 2: the figures show above show the values of saturation at a particular point in the mesh-see figure (a), (a) location of the point (b) saturation values of the point (continued) (see online version for colours)

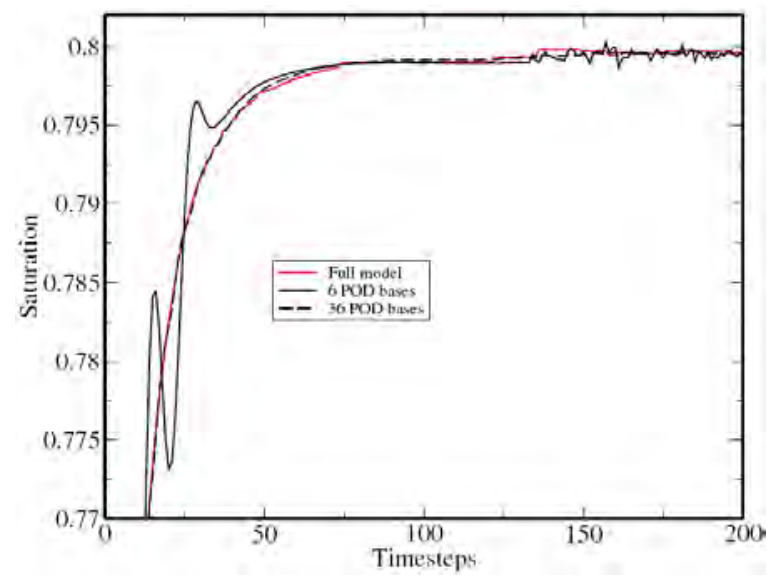

(b)

Evaluation of accuracy of the POD model was carried out through comparison of POD solutions with those from the full model. Figure 11 shows the saturation solutions obtained from the high fidelity full model and NIROM with six and 36 POD bases at time instances 500 days and 800 days. It shows clearly that the results of NIROM are close to that of high fidelity full model. The solutions of saturation at a particular point [Figure 12 (a)] in the computational domain are presented in Figure 12. The figure again shows that the accuracy of solution can be improved by increasing the number of POD bases functions to 36 and the NIROM using 36 POD bases is in good agreement with the high fidelity full model.

The error of saturation solutions between the high fidelity full model and the NIROMs with six and 36 POD bases are presented in Figure 13.

\subsection{Efficiency of the NIROM}

The CPU cost of NIROM includes online cost and offline cost. The offline cost includes the time of forming basis functions and hypersurfaces, which is pre-computed. Table 1 shows the online CPU cost required for the high fidelity full model and NIROM. The simulations were performed on a machine with 12 cores. The CPU model is Intel(R) Xeon(R) X5680 and each core has a frequency of $3.3 \mathrm{GHz}$. The RAM has a memory of $48 \mathrm{~GB}$. The test cases were run in serial, which means only one core was used for the simulation. The offline CPU time required for constructing the POD bases and the interpolation function $f_{\alpha, v, j}, f_{\alpha, p, j}$ and $f_{\alpha, S, i}$ (see algorithm 1) is not listed here. The online CPU time for running the NIROM includes:

- interpolation for calculating the POD coefficients $\mathbf{v}_{r, \alpha, j}^{n}, \mathbf{p}_{r, \alpha, j}^{n}$ and $\mathbf{S}_{r, \alpha, j}^{n}$ [see equation (15)]

- projecting the $\mathbf{v}_{r, \alpha, j}^{n}, \mathbf{p}_{r, \alpha, j}^{n}$ and $\mathbf{S}_{r, \alpha, j}^{n}$ onto the full space [see equation (7)]. 
The online CPU cost required for the NIROM is considerably less than that for the full model and is reduced by a factor of 2,500. It is worth noting that as the number of nodes increases the CPU time required for the full model increases rapidly while the CPU time for the NIROM almost remains the same.

Figure 13 Case 2: the figures show the error of saturation between the full model and NIROM of the 3D fluvial channel problem at time instances 500 days and 800 days using 6 and 36 POD bases, (a) error of NIROM with 6 pod bases, $t=500$ days (b) error of NIROM with 6 POD bases, $t=800$ days (c) error of NIROM with 36 POD bases, $t=500$ days $(d)$ error of NIROM with 36 POD bases, $t=800$ days (see online version for colours)

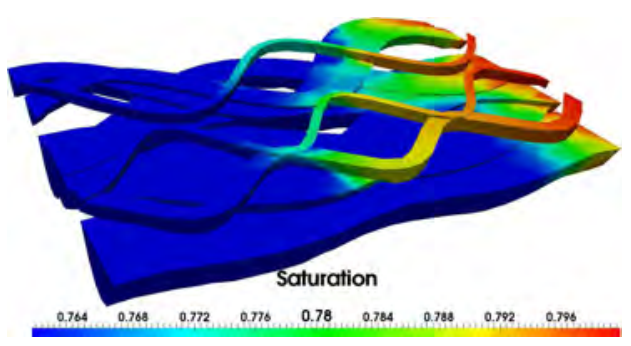

(a)

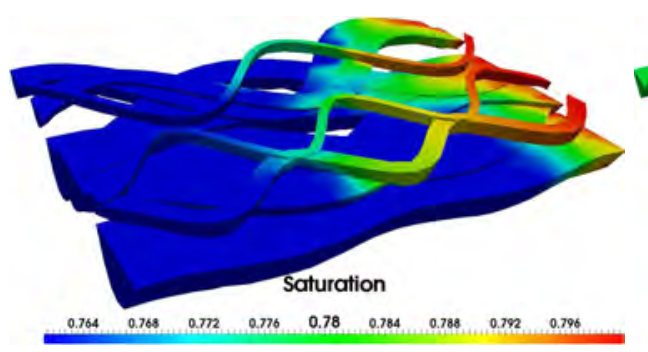

(c)

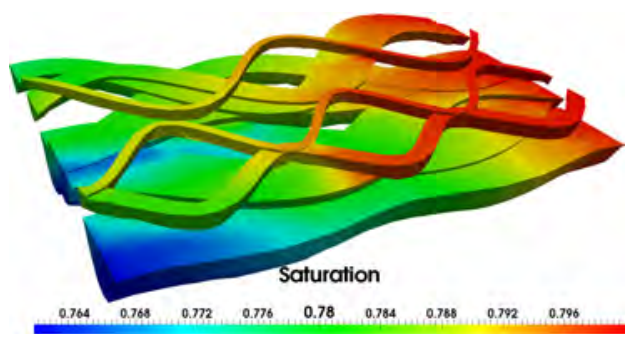

(b)

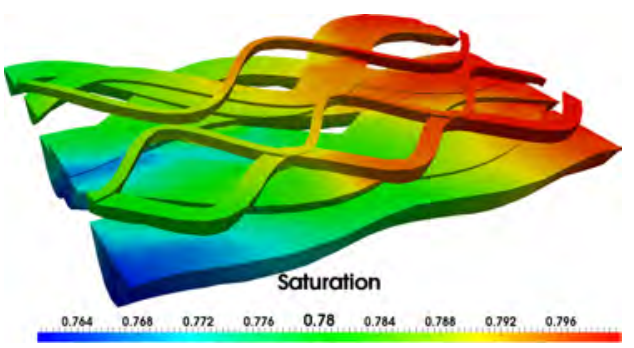

(d)

Table 1 Comparison of the online CPU time (dimensionless) required for running the full model and NIROM during one time step

\begin{tabular}{lccccc}
\hline Cases & Model & Assembling and solving & Projection & Interpolation & Total \\
\hline Case 1 & Full model & 0.81605 & 0 & 0 & 0.81605 \\
& NIROM & 0 & 0.0003 & 0.0001 & 0.00040 \\
Case 2 & Full model & 98.3998 & 0 & 0 & 98.3998 \\
& NIROM & 0 & 0.0003 & 0.0001 & 0.00040 \\
\hline
\end{tabular}

\section{Conclusions}

A NIROM based on hypersurfaces representation has recently been developed for IC-FERST which has the capabilities of using 
1 anisotropic unstructured meshes to resolve fine scale flow feature

2 a novel control volume finite element method to resolve the high-order flux flows on CV boundaries.

In this work the NIROM has been further applied to a fluvial channel problem.

A RBF interpolation method is used to form a multi-dimensional interpolation function (hyper surface) that represents the solution of the 3D reservoir model within the reduced space. The non-intrusive approach used here to construct the NIROM is generic and does not require any information of the original source code or the model equations. It can be applied to any software or commercial codes. In addition, it avoids the instability of existing Galerkin POD ROMs (Walton et al., 2013), the results might be smoothed by RBF (Myers, 1999).

The capabilities of the newly developed NIROM are illustrated in two test cases: a simple horizontally layered porous media case and a complicated 3D fluvial channel case. A comparison between the full and NIROM results are made. An error analysis was also carried out for the validation and accuracy assessment of the NIROM. It is shown that the NIROM exhibits an overall good agreement with the high fidelity full model. An increase in the number of POD bases leads to an improvement in the accuracy of the NIROM. The saturation shock-front can be captured with relatively few POD basis functions, 18 POD basis function [Figure 4(h)] in the examples.

In comparison to the full model, without compromising the accuracy of results the CPU time required for the NIROM can be reduced by a factor of 2,500. It is worth pointing out that for $3 \mathrm{D}$ large scale reservoir simulation, an increase in the number of nodes used in the computational domain will result in a large increase of the CPU time in the full simulation, but has very little effect on that of the NIROM. Future work will investigate the effects of applying this new approach to more complex 3D reservoir simulation cases and extend this method for varying parameters reservoir simulation problems. It is also interesting to apply this method to complicated fracture problems, for example, the work demonstrated in Yang $(2016,2018)$.

\section{Acknowledgements}

The authors are grateful for the support of the EPSRC grant: Managing Air for Green Inner Cities (MAGIC) (EP/N010221/1), EPSRCMEMPHIS multi-phase flow programme grant (EP/K003976/1), NSFC grant 11502241, EPSRC (Smart-Geo Wells grant EP/R005761/1), funding from the European Union Seventh Frame work Programme (FP7/20072013) under grant agreement No. 603663 for the research project PEARL (Preparing for Extreme And Rare events in coastal regions). No data was generated in the course of this work. For further information, please contact the corresponding author at (dh.xiao@imperial.ac.uk)or the AMCG Group (http://www.imperial.ac.uk/earth-science/ research/researchgroups/amcg/) as required. 


\section{References}

Altaf, M.U. (2011) Model Reduced Variational Data Assimilation for Shallow Water Flow Models, $\mathrm{PhD}$ thesis, Delft University of Technology.

Audouze, C., De Vuyst, F. and Nair, P.B. (2009) 'Reduced-order modeling of parameterized PDES using time-space-parameter principal component analysis', International Journal for Numerical Methods in Engineering, Vol. 80, No. 8, pp.1025-1057.

Audouze, C., Vuyst, F.D. and Nair, P.B. (2013) 'Nonintrusive reduced-order modeling of parametrized time-dependent partial differential equations', Numerical Methods for Partial Differential Equations, Vol. 29, No. 5, pp.1587-1628.

Barrault, M., Maday, Y., Nguyen, N.C. and Patera, A.T. (2004) 'An empirical interpolation method: application to efficient reduced-basis discretization of partial differential equations', C. R. Acad. Sci. Paris, Ser, Vol. 339, No. 9, pp.667-672.

Bou-Mosleh, C., Carlberg, K. and Farhat, C. (2011) 'Efficient non-linear model reduction via a least-squares Petrov-Galerkin projection and compressive tensor approximations', International Journal for Numerical Methods in Engineering, Vol. 86, No. 2, pp.155-181.

Cao, Y., Zhu, J., Navon, I.M. and Luo, Z. (2007) 'A reduced order approach to four dimensional variational data assimilation using proper orthogonal decomposition', International Journal for Numerical Methods in Fluids, Vol. 53, No. 10, pp.1571-1583.

Cardoso, M.A. (2009) Development and Application of Reduced-Order Modeling Procedures for Reservoir Simulation, ProQuest Dissertations Publishing, Stanford University, Cardoso, Marco Antonio.

Cardoso, M.A., Durlofsky, L.J. and Sarma, P. (2009) 'Development and application of reducedorder modeling procedures for subsurface flow simulation', International Journal for Numerical Methods in Engineering, Vol. 77, No. 9, pp.1322-1350.

Carlberg, K., Farhat, C., Cortial, J. and Amsallem, D. (2013) 'The GNAT method for nonlinear model reduction: effective implementation and application to computational fluid dynamics and turbulent flows', Journal of Computational Physics, Vol. 242, No. 1, pp.623-647.

Chaturantabut, S. and Sorensen, D.C. (2010) 'Nonlinear model reduction via discrete empirical interpolation', SIAM J. Sci. Comput., Vol. 32, No. 5, pp.2737-2764.

Chaturantabut, S. and Sorensen, D.C. (2011) 'Application of POD and DEIM on dimension reduction of non-linear miscible viscous fingering in porousmedia', Mathematical and Computer Modelling of Dynamical Systems, Vol. 17, No. 4, pp.337-353.

Chen, X., Akella, S. and Navon, I.M. (2012) 'A dual-weighted trust-region adaptive POD 4-D var applied to a finite-volume shallow water equations model on the sphere', International Journal for Numerical Methods in Fluids, Vol. 68, No. 3, pp.377-402.

Chen, X., Navon, I.M. and Fang, F. (2011) 'A dual-weighted trust-region adaptive POD 4D-VAR applied to a finite-element shallow-water equations model', International Journal for Numerical Methods in Fluids, Vol. 65, No. 5, pp.520-541.

Daescu, D.N. and Navon, I.M. (2008) 'A dual-weighted approach to order reduction in 4D-var data assimilation', Monthly Weather Review, Vol. 136, No. 3, pp.1026-1041.

Du, J., Fang, F., Pain, C.C., Navon, I.M., Zhu, J. and Ham, D.A. (2013) 'POD reduced-order unstructured mesh modeling applied to 2D and 3D fluid flow', Computers and Mathematics with Applications, Vol. 65, No. 3, pp.362-379.

Fang, F., Pain, C., Navon, I.M., Elsheikh, A.H., Du, J. and Xiao, D. (2013) 'Non-linear PetrovGalerkin methods for reduced order hyperbolic equations and discontinuous finite element methods', Journal of Computational Physics, Vol. 234, No. 1, pp.540-559. 
Fang, F., Pain, C.C., Navon, I.M., Gorman, G.J., Piggott, M.D., Allison, P.A. and Goddard, A.J.H. (2010) 'A POD goal-oriented error measure for mesh optimization', International Journal for Numerical Methods in Fluids, Vol. 63, No. 2, pp.185-206.

Fang, F., Pain, C.C., Navon, I.M., Piggott, M.D., Gorman, G.J., Allison, P.A. and Goddard, A.J.H. (2009) 'Reduced-order modelling of an adaptive mesh ocean model', International Journal for Numerical Methods in Fluids, Vol. 59, No. 8, pp.827-851.

Fang, F., Zhang, T., Pavlidis, D., Pain, C.C., Buchan, A.G. and Navon, I.M. (2014) 'Reduced order modelling of an unstructured mesh air pollution model and application in 2D/3D urban street canyons', Atmospheric Environment, Vol. 96, No. 1, pp.96-106.

Franca, L.P. and Frey, S.L. (1992) 'Stabilized finite element methods: Ii. The incompressible Navier-Stokes equations', Computer Methods in Applied Mechanics and Engineering, Vol. 99, Nos. 2-3, pp.209-233.

Fukunaga, K. (1990) Introduction to Statistical Recognition, 2nd ed., pp.5-33, Computer Science and Scientific Computing Series, Academic Press, Academic Press, Boston, MA.

Ghasemi, M., Ibrahim, A., Gildin, E. et al. (2014) 'Reduced order modeling in reservoir simulation using the bilinear approximation techniques', in SPE Latin America and Caribbean Petroleum Engineering Conference, Society of Petroleum Engineers.

Han, C. (2012) Blackbox Stencil Interpolation Method for Model Reduction, Master's thesis, Massachusetts Institute of Technology.

Heijn, T., Markovinovic, R. and Jansen, J-D. (2004) 'Generation of low-order reservoir models using system-theoretical concepts', SPE Journal, Vol. 9, No. 2, pp.202-218.

Jafarpour, A. and Sabetghadam, F. (2012) 'Regularization of the POD-Galerkin dynamical systems of the Kuramoto-Sivashinsky equation', Applied Mathematics and Computation, Vol. 218, No. 10, pp.6012-6026.

Jolliffe, I.T. (2002) Principal Component Analysis, 2nd ed., pp.559-572, Springer, Aberdeen, UK.

Klie, H. et al. (2013) 'Unlocking fast reservoir predictions via nonintrusive reduced order models', in SPE Reservoir Simulation Symposium, Society of Petroleum Engineers.

Myers, D.E. (1999) 'Smoothing and interpolation with radial basis functions', Boundary Element Technology, Vol. 13, No. 1, pp.365-376.

Pearson, K. (1901) 'On lines and planes of closest fit to systems of points in space', Philosophical Magazine, Vol. 2, No. 11, pp.559-572.

Salinas, P., Percival, J.R., Pavlidis, D., Xie, Z., Gomes, J., Pain, C.C. and Jackson, M.D. (2015) ‘A discontinuous overlapping control volume finite element method for multi-phase porous media flow using dynamic unstructured mesh optimization', in SPE 173279.

Serpas, M., Chu, Y. and Hahn, J. (2011) 'State-preserving nonlinear model reduction procedure', Chemical Engineering Science, Vol. 66, No. 17, pp.3907-3913.

Stefanescu, R. and Navon, I.M. (2013) 'POD/DEIM nonlinear model order reduction of an ADI implicit shallow water equations model', Journal of Computational Physics, Vol. 237, No. 1, pp.95-114.

Stefanescu, R., Sandu, A. and Navon, I.M. (2014) 'Comparison of POD reduced order strategies for the nonlinear 2D shallow water equations', International Journal for Numerical Methods in Fluids, Vol. 76, No. 8, pp.497-521.

Su, K., Latham, J-P., Pavlidis, D., Xiang, J., Fang, F., Mostaghimi, P., Percival, J.R., Pain, C.C. and Jackson, M.D. (2015) 'Multiphase flow simulation through porous media with explicitly resolved fractures', Geofluids, November 2015, Vol. 15, No. 4, pp.592-607.

Walton, S., Hassan, O. and Morgan, K. (2013) 'Reduced order modelling for unsteady fluid flow using proper orthogonal decomposition and radial basis functions', Applied Mathematical Modelling, Vol. 37, No. 20, pp.8930-8945.

Willcox, K. (2006) 'Unsteady flow sensing and estimation via the gappy proper orthogonal decomposition', Computers \& Fluids, Vol. 35, No. 2, pp.208-226. 
Willcox, K. and Megretski, A. (2003) 'Model reduction for large-scale linear applications', in Proc. of 13th IFAC Symposium on System Identification, Rotterdam, Netherlands, pp.1431-1436.

Xiao, D., Fang, F., Buchan, A.G., Pain, C.C., Navon, I.M. and Muggeridge, A. (2015b) 'Nonintrusive reduced order modelling of the Navier-Stokes equations', Computer Methods in Applied Mechanics and Engineering, Vol. 293, No. 1, pp.552-541.

Xiao, D., Fang, F., Buchan, A.G., Pain, C.C., Navon, I.M., Du, J. and Hu, G. (2014) 'Nonlinear model reduction for the Navier-Stokes equations using residual DEIM method', Journal of Computational Physics, Vol. 263, pp.1-18.

Xiao, D., Fang, F., Du, J., Pain, C.C., Navon, I.M., Buchan, A.G., ElSheikh, A.H. and Hu, G. (2013) 'Non-linear Petrov-Galerkin methods for reduced order modelling of the Navier-Stokes equations using amixed finite element pair', Computer Methods In Applied Mechanics and Engineering, Vol. 255, No. 1, pp.147-157.

Xiao, D., Fang, F., Pain, C. and Hu, G. (2015a) 'Non-intrusive reduced order modelling of the Navier-Stokes equations based on RBF interpolation', International Journal for Numerical Methods in Fluids, Vol. 79, No. 11, pp.580-595.

Xiao, D., Fang, F., Pain, C.C. and Navon, I.M. (2017b) 'Towards non-intrusive reduced order 3D free surface flow modelling', Ocean Engineering, Vol. 140, No. 1, pp.155-168.

Xiao, D., Yang, P., Fang, F., Xiang, J., Pain, C.C. and Navon, I.M. (2016) 'Non-intrusive reduced order modelling of fluid structure interactions', Computer Methods in Applied Mechanics and Engineering, Vol. 303, No. 1, pp.35-54.

Xiao, D., Yang, P., Fang, F., Xiang, J., Pain, C.C., Navon, I.M. and Chen, M. (2017a) 'A nonintrusive reduced-ordermodel for compressible fluid and fractured solid coupling and its application to blasting', Journal of Computational Physics, Vol. 330, No. 1, pp.221-244.

Yang, Y., Efendiev, Y., Ghasemi, M., Gildin, E., Calo, V.M. et al. (2015) 'Fast multiscale reservoir simulations using POD-DEIM model reduction', in SPE Reservoir Simulation Symposium, Society of Petroleum Engineers.

Yang, Y., Tang, X., Zheng, H., Liu, Q. and He, L. (2016) 'Three dimensional fracture propagation with numerical manifold method', Engineering Analysis with Boundary Elements, Vol. 72, No. 1, pp.65-77.

Yang, Y., Tang, X., Zheng, H., Liu, Q. and Liu, Z. (2018) 'Hydraulic fracturing modeling using the enriched numerical manifold method', Applied Mathematical Modelling, Vol. 53, No. 1, pp.462-486.

Yoon, S., Alghareeb, Z., Williams, J. et al. (2014) 'Development of reduced order oil reservoir models using localized DEIM', in SPE Annual Technical Conference and Exhibition, Springer US, pp.1-26. 